\title{
Psychometric Properties of Screening Questionnaires To Detect Depression In Primary Healthcare Setting In Rural Ethiopia
}

\section{Kassahun Habtamu ( $\sim$ kasshabmek@gmail.com )}

Addis Ababa University

Rahel Berhane

Addis Ababa University

Girmay Medhin

Addis Ababa University

Charlotte Hanlon

Addis Ababa University

Abebaw Fekadu

Addis Ababa University

\section{Research Article}

Keywords: Depression, Patient health questionnaire, Primary healthcare, Psychometric properties, Convergent validity, Construct validity, Screening, Rural Ethiopia

Posted Date: December 28th, 2021

DOI: https://doi.org/10.21203/rs.3.rs-1163798/v1

License: (c) (1) This work is licensed under a Creative Commons Attribution 4.0 International License. Read Full License 


\section{Abstract}

Background: Much of the research about the validity of depression screening questionnaires is on criterion validity. Evidence is scarce on the concurrent, convergent and construct validity of these measures, particularly from low-income countries. This study aimed to evaluate the psychometric properties of depression screening questionnaires in primary healthcare $(\mathrm{PHC})$ in rural Ethiopia.

Methods: A facility-based cross-sectional study was conducted with 587 participants recruited from patients attending three PHC facilities and two Holy water sites. The psychometric properties of five mental health screening questionnaires were evaluated: the nine item Patient Health Questionnaire (PHQ9), the two item version of PHQ-9 (PHQ-2), a version of PHQ-9 with two added items of irritability and noise intolerance (PHQ-11), the Patient Health Questionnaire-15 (PHQ-15), and the World Health Organization-Five Well-being Index (WHO-5). Clinical diagnosis of depression was ascertained by psychiatrists using a semi-structured interview. We analyzed data using exploratory factor analysis, Spearman's rank order correlation coefficient (Rho), the Mann Whitney test of the equality of medians, univariate logistic regression and Cronbach's alpha.

Results: PHQ-9, PHQ-11 and WHO-5 were found to be unidimensional, with items in each scale highly loading onto one factor (factor loadings ranging from 0.64 to 0.87 ). The items of each instrument were internally consistent, with Cronbach's alpha ranging from 0.72 (PHQ-2) to 0.89 (PHQ-11). Scores for all screening scales were moderately or highly correlated with each other (Rho $=0.58$ to 0.98 ) and moderately correlated with anxiety and disability scores. Median scores of all screening scales were significantly higher in those diagnosed with depression. The association of items measuring emotional and cognitive symptoms with the diagnosis of depression was stronger than the association with items measuring somatic symptoms. Irritability and noise intolerance had higher association with depression diagnosis than PHQ-9 items.

Conclusion: Emotional and cognitive symptoms are more useful than somatic symptoms to predict the diagnosis of depression. Irritability and noise intolerance are more useful to detect depression. Future research should focus on testing the unidimensionality of PHQ-9, PHQ-11 and WHO-5 using confirmatory factor analysis; establishing the criterion validity of PHQ-11 and WHO-5; and on assessing test-retest reliability of all the measures.

\section{Background}

Depression is the leading cause of disability, as measured by Years Lived with Disability [1], and projected to become the second leading contributor to the global burden of disease by 2030 [2]. Depression is associated with increased use of healthcare resources [3] and results in an enormous economic burden [4] as it is one of the most commonly occurring illnesses and leads to substantial loss in productivity. Depression is associated with elevated morbidity and mortality related to suicide [5]; it is often co-morbid with other physical as well as mental health conditions [6]. The prevalence of depression in primary 
healthcare (PHC) is higher than in the general population [7]. Around $10 \%$ of all primary care visits are depression related and most patients who have depression get treatment by PHC clinicians [8].

Despite a high prevalence of depression in primary care [7], underrecognition is a major challenge globally [9]. In high-income countries, approximately $50 \%$ of primary care doctors correctly identify individuals with depression, and only $34 \%$ record it in their notes [10]. In low and middle-income countries (LMICs), the detection of depression in the PHC setting is extremely low. For instance, a study conducted in Ethiopia showed that less than $5 \%$ of patients presenting to primary healthcare with potential depression received a clinical diagnosis of depression [8]. Another study conducted in a health center in Malawi found a zero percent depression detection rate by primary healthcare workers [11]. Low levels of detection of depression are jeopardizing the impact of efforts to scale up integration of mental healthcare into PHC [8].

Screening with self-reported questionnaires is considered to be a potentially useful approach to aid PHC clinicians in recognizing patients who may have depression [12]. Guidelines developed in some high income countries recommend routine screening for depression in PHC [13]. A systematic review found that screening questionnaires are likely to be effective in improving recognition of depression when they are used in conjunction with other interventions [14]. However, screening alone does not seem to be effective; it has to be accompanied by disclosure of screening results to the clinician, training of the clinical and other relevant staff, supportive supervision and clear referral pathways $[15,16]$. Nevertheless, studies on the utility of screening questionnaires to increase detection of depression in real-world settings are mostly from high-income countries [17] and evidence is scarce whether these instruments are useful in LMICs [14]. In low-income countries, depression screening tools may have effect to improve PHC providers' diagnosis of depression [18]; and this needs the use of brief and psychometrically sound screening instruments.

A number of brief screening questionnaires that can be used to screen for depression in the PHC setting do exist $[14,17]$. However, the bulk of evidence regarding the validity and reliability of these questionnaires is from high income countries [19]. There are some studies on the validity of depression screening tools from LMICs, but much of the evidence is on criterion validity [20]. There is only limited research on the convergent and construct validity of depression screening tools, particularly in lowincome country settings. There are a few studies conducted in Ethiopia on the criterion validity of depression screening tools, particularly on the nine item Patient Health Questionnaire (PHQ-9) [18, 2123]. Only one of these validation studies was conducted in the PHC setting and validated more than one depression screening tool [18]. Moreover, almost all of the studies focused on criterion validity of the tools against clinician diagnosis of depression. None of the studies investigated the factor structure of the screening tools and which of the symptoms or items in the scales are associated with diagnosis of depression ascertained by psychiatrists.

This study, therefore, aimed to evaluate the concurrent, convergent and construct validity of depression screening questionnaires in PHC setting in rural Ethiopia. Specifically, we sought to determine i) the factor 
structure of depression screening questionnaires using exploratory factor analysis ii) convergent and concurrent validity of the instruments by computing the correlation of aggregate scores of each screening tool with other depression measures and measures of other variables which are theoretically known to have correlation with depressive symptoms iii) known group differences by comparing median depressive symptom scores between those who have psychiatrist diagnosis of depression and those who do not have depression diagnosis iv) association of each item of the depression screening tools with psychiatrist depression diagnosis.

\section{Methods}

\section{Study design}

A facility-based cross-sectional study was conducted to investigate the psychometric properties of brief depression screening questionnaires when they are used in PHC setting in rural Ethiopia.

\section{Study setting and context}

The study was conducted in Sodo district, Gurage Zone, Southern Nations, Nationalities and Peoples Region (SNNPR). The district is predominantly rural, and is located $100 \mathrm{~km}$ south of Addis Ababa, the capital city of Ethiopia. The population of the district at the time of the study was estimated to be 161,952 (79,356 men and 82,596 women) living in 58 sub-districts [24]. The largest ethnic group in the district is Sodo Gurage (85.3\%), Amharic is the official language [8] and $97 \%$ of the population are followers of Orthodox Christian [24].

The district has one primary hospital, 8 health centers and 58 health posts [25]. Staff in health centers constituted nurses, health officers, and midwives, who are trained at degree or diploma level. Health centers provide primary care for about 20,000 (rural areas) to 40,000 (urban areas) people; whereas each health post serves 3000-5000 people. Healthcare providers in the health centers and health posts deliver services such as diagnosis and treatment of communicable diseases (e.g. malaria, tuberculosis, and water-borne diseases), family planning, antenatal care, malaria prevention, and give advice on the effects of harmful traditional practices and sanitation. At the time of this study, efforts had been made to integrate mental health services into the primary care level as part of the PRogramme for Improving Mental Healthcare (PRIME) project [26]. Before the start of the PRIME project, people with mental disorders had to travel to an outpatient clinic in Butajira town, which is led by psychiatric nurses; for inpatient psychiatric treatment or interventions for substance use disorders, they had to travel to Addis Ababa [24].

This study was carried out as part of the Improving Detection of depression in primary care in SubSaharan Africa (IDEAS) project [27]. The IDEAS study aimed to develop and evaluate interventions that would help improve the recognition of depression in the PHC setting in Ethiopia. To develop this paper, we used the baseline data collected for the IDEAS cohort study.

\section{Participants and recruitment}


A total of 1363 consecutive patients attending three PHC facilities and two Holy water sites in the Sodo district were invited to participate in the study. Of these, 587 participants fulfilled the inclusion criteria and gave consent to be included in the study (Figure 1). Participant recruitment was done in two phases. In phase 1, 410 participants were recruited from 29 August 2019 to 17 March 2020; whereas 177 were recruited in phase 2 from 2 December 2020 to 18 February 2021. Patients were approached after they had consulted the PHC provider. Patients were recruited in to the study if they were adults (age $\geq 18$ ), were able to speak and understand Amharic (the official language in Ethiopia), and gave informed consent.

\section{Brief screening questionnaires for depression}

The brief depression screening tools considered in this study included the nine item Patient Health Questionnaire (PHQ-9) [28], the two item version of the patient health questionnaire ( $\mathrm{PHQ}-2)$, the nine item PHQ with two additional contextually relevant items (PHQ-11), the Patient Health Questionnaire-15 (PHQ15) [29] and the World Health Organization-Five Well-being Index (WHO-5) [30].

\section{1) Patient health questionnaire (2-item, 9-item and 11 item versions)}

The PHQ-9 is a nine item scale that was developed and validated as a depression assessment tool [28]. It has been widely used in clinical and population-based studies worldwide as a screening instrument [31]. The PHQ-9 follows the Diagnostic and Statistical Manual of Mental Disorders, 4th edition (DSM-IV) diagnostic criteria for a depressive episode [32] and asks about symptoms present in the preceding two weeks. Each question in the PHQ-9 is rated from 0 (not at all) to 3 (nearly every day). The response categories indicate the amount of time that the symptom was present. Responses for each item can be summed, which gives a total symptom score ranging from 0 to 27 . The DSM diagnostic criteria for a major depressive episode can also be applied to give a possible diagnosis of depression using PHQ-9 [33]. The criterion validity of the PHQ-9 as a screening, but not diagnostic, tool has been demonstrated in Ethiopia in the PHC setting [18] and at a referral hospital in Addis Ababa [21]. A study was also conducted in Ethiopia on the criterion validity of the PHQ-9 among cancer patients attending the oncology clinic at a specialized hospital [22].

The PHQ-2 includes the first two items of the PHQ-9 and is usually used as an initial depression screening instrument for major depressive disorder [34]. The PHQ-2 has been found to be a useful screening tool in PHC settings, particularly in high-income countries [35]. Questions on the PHQ-2 have the same response categories as the PHQ-9 and ask about frequency of symptoms over the preceding two weeks. Responses on the PHQ-2 can be summed and scores range from zero to six. A study conducted in a PHC setting in Ethiopia showed that PHQ-2 has lower validity than the PHQ-9 [18]. The PHQ-11 includes all the items in the PHQ-9 and two new additional items (irritability and noise intolerance), which were found to be relevant expressions of depression in the Ethiopian context through qualitative studies and clinical experiences. A previous validation study in Ethiopia [18] found that $50 \%$ of the true cases of major depressive disorder had irritability.

\section{2) Patient health questionnaire-15 (PHQ-15)}


PHQ-15 is a brief, self-administered questionnaire that is used to assess somatic symptom severity and screen for the potential presence of somatisation and somatoform disorders based on the DSM-IV criteria in adults [29]. The PHQ-15 assesses the presence and severity of 15 common somatic symptoms in primary health care, such as fatigue in the last four weeks. The response categories for the PHQ-15 are "not bothered at all" (0), "bothered a little" (1) and "bothered a lot" (2) [36]. Aggregate scores range from 0 to 30 with higher scores indicating higher symptom severity. It has well-established psychometric properties, is available in multiple languages and has been recommended for use in large-scale studies [37].

In a recent systematic review [38], PHQ-15 had very high internal consistency, test-retest reliability, structural validity, and construct validity, as well as good criterion validity. Depression is less frequently diagnosed, particularly in non-Western settings, including in $\mathrm{PHC}$, which may be due to somatization of depressive symptoms [39]. A somatic symptom-focused screening tool may be useful in detecting depression in these settings. Nevertheless, studies on the potential use of the PHQ-15 to screening depression in PHC are scarce. A study conducted among Korean College and graduate students showed that the PHQ-15 can be used as an effective screening tool for depression in that setting [40].

\section{3) World Health Organization-Five Well-being Index (WHO-5)}

The five item World Health Organization Well-Being Index (WHO-5) is a short and generic questionnaire which measures subjective well-being [30]. It consists of simple and non-invasive questions and the respondents are asked to rate how well each of the five statements apply to them over the last two weeks [41]. Each of the five items is scored from 5 (all of the time) to 0 (none of the time), with aggregate score ranging from 0 to 25 . The WHO-5 has been translated into over 30 languages and has been used in research projects all over the world [42]. The tool is used widely for screening depression in the PHC setting [34].

A systematic review showed that the WHO-5 has adequate validity both as a screening tool for depression and as an outcome measure in clinical trials [42]; it has high criterion validity and is sensitive and specific as a screening tool for depression [41]. The review further found that the WHO-5 had a very high negative association with self- and observer-rated measures of depressive symptoms. However, the criterion validity of the WHO-5 has not been determined in the African setting. We found only one study which investigated the construct, discriminant and convergent validity of the scale in rural Kenya [43].

\section{Other measures}

We used the seven item generalized anxiety disorder scale (GAD-7) to measure anxiety [44]. The GAD-7 has been validated both in PHC setting and the general population [45]. It measures how often the respondent has been bothered by seven symptoms of anxiety during the last two weeks with four response options ranging from 0 (not at all) to 3 (nearly every day). The scale has good sensitivity and specificity for the diagnosis of the most common anxiety disorders in primary care [46]. Overall, the GAD7 is found to be a valid and efficient tool for detecting anxiety disorders [44]. The 12 item version of the 
World Health Organization Disability Assessment Schedule (WHODAS-2.0) [47] was used to assess functional impairment. The measure is considered to have cross-cultural validity [48] and the Amharic version has been validated in Ethiopia in a sample of people with severe mental disorders [49].

The Oslo social support scale (OSSS-3) was used to measure general social support. OSSS-3 is a three item scale which asks about number of close confidants, sense of concern or interest from other people and ease of getting practical help from neighbors [50]. OSSS-3 is a feasible instrument and has good predictive validity and convergent validity [51]. It has been used in previous community and facility-based studies in Ethiopia and demonstrated good utility [24]. The List of Threatening Experiences (LTE) was used to collect data on participants' experience of stressful life events [52]. The LTE measures the occurrence of 12 prevalent major stressful events (e.g. death of a close relative or friend, loss of relationship, imprisonment and being the victim of theft) in the preceding 6 months, with dichotomous responses (yes/no). It has been adapted and used in a rural Ethiopian setting [24]. We developed and administered a structured self-report demographic and socio-economic characteristics questionnaire to collect data on such variables as sex, age, urban-rural residence, religion, marital status, educational status, and socio-economic status of the participants.

\section{Procedure}

We used the Amharic version of the PHQ-9 which has already been adapted by Hanlon et al [18]; the study also established the semantic, technical and content validity of the scale in the rural Ethiopian setting. The PHQ-15 and WHO-5 scales were translated into Amharic independently by two Ethiopian mental health researchers and then back-translated into English by two other researchers who are familiar with the study setting. We produced the final versions of these instruments through expert consensus. Changing the scales from self-completed to interviewer-administered required us to make some minor modifications as it has been done in previous studies from low-income countries [53].

Data for this study were collected by lay-data collectors and psychiatrists. Lay-data collectors administered the brief screening tools and other structured questionnaires. For those who obtained scores above the locally validated cut-off points in one of the depression screening scales and a random sample of screen negatives, clinical diagnosis of depression was done by Ethiopian psychiatrists. A semistructured version of the depression module of the Mini-International Neuropsychiatric Interview (MINI) was used for clinical diagnosis by psychiatrist. The administration of the brief depression screening tools preceded the psychiatrist assessment. Psychiatrists were masked to the results of the screening questionnaires. All the lay-interviewers have at least diploma level training and have many years of experience in data collection related to mental health research. Senior mental health researchers trained the lay-data collectors for five days, which included role plays and observed pilot interviews. The psychiatrists who did the clinical assessment were trained by another senior Ethiopian psychiatrist for two days in the administration of the MINI. The training included role play and piloting of the clinical assessment.

\section{Data management and analysis}


Data were checked for completeness in the field by research assistants and supervisors. Data were double entered with consistency checks in Epidata version 4.2.0. Data entry was done on the day of data collection where possible. Data cleaning was done using frequency distributions and logic checks, with reference to source documents as required. We analyzed the data using Stata version 14 . Frequencies and percentages were used to summarize variables which were categorical, whereas continuous variables were summarized using mean and standard deviation.

We did exploratory factor analysis to investigate the construct validity of each of the brief depression screening questionnaires (except PHQ-2) with principal axis factoring method and we applied varimax rotation. Both Eigenvalues and scree plots were considered to determine the number of factors to be retained as indicators of scale dimensions. Rotated factor loadings were reported as indicators of the association of each item with the underlying factor. Concurrent and convergent validity were evaluated using non-parametric tests. Spearman's rank order correlation coefficient (Rho) was computed for the association among the scores of the brief depression screening tools and the scores of each of the brief depression screening tools and scores of GAD-7, WHODAS-2, OSS-3 and LTE. The Mann Whitney test of the equality of medians was used to compare the distribution of each of the depression screening scale scores in people who have been diagnosed to have depression and in those who have not been diagnosed to have depression. Univariate logistic regression analysis was carried out to explore the association of each item in the depression screening tools with psychiatrist depression diagnosis. This was done to identify symptoms that are potentially useful to detect depression in the PHC setting. Internal consistency of items in each of the depression screening questionnaires was evaluated using Cronbach's alpha.

\section{Ethical considerations}

The study was conducted in accordance with the Declaration of Helsinki. The study protocol was reviewed and ethical approval was obtained from the Institutional Review Board of the College of Health Sciences, Addis Ababa University (Reference Number 007/18/Psy). Written informed consent was obtained from all the participants after the nature of the study and the information sought had been fully explained. Non-literate participants gave finger-prints to signify their willingness to participate. Study participants who were identified by the psychiatrists as having a mental health condition were provided with the appropriate treatment and follow-up in the out-patient psychiatric clinic in Bui primary hospital.

\section{Results}

\section{Characteristics of study participants}

We included a total of 587 adults into the study and all of these participants had complete data. A little more than half of the participants were men (51.3\%). The mean age of the participants was 35.76 (SD= 13.8), and a quarter were not literate (26.1\%). The majority of the participants were married $(72.8 \%)$ and came from rural areas $(69.1 \%)$. More than $95 \%$ of the participants were Christian. In terms of occupation, $38.7 \%$ were farmers and $26.2 \%$ were housewives. The main reason for their visit for the majority of the 
participants $(74.1 \%)$ was new illness and a little more than a quarter of the participants $(26.9 \%)$ had ever sought help in the facility or other places for their presenting complaint. See Table 1 for details of the socio-demographic characteristics of the participants.

Table 1: Socio-demographic characteristics of participants $(n=587)$

$S D=$ Standard deviation $; n=$ Number of participants

Construct validity

Exploratory factor analysis indicated, with both eigenvalue and scree plot criteria, that the PHQ-9, PHQ-11 and WHO-5 were unidimensional, with the factor in each scale explaining $51.0 \%, 48.3 \%$ and $69.4 \%$ of the total variance, respectively. All items in the scales highly loaded onto the resulting factor. The factor loadings for the PHQ-9 ranged from 0.65 to 0.79 ; for PHQ-11 0.64 to 0.77 ; and for WHO-5 0.78 to 0.87 . See Table 2.

Table 2: Rotated factor loadings of the exploratory factor analysis of PHQ-9, PHQ-11 and WHO-5 


\begin{tabular}{|c|c|c|c|}
\hline Characteristics & & $\mathbf{N}$ & $\%$ \\
\hline \multirow[t]{2}{*}{ Sex } & Female & 286 & 48.7 \\
\hline & Male & 301 & 51.3 \\
\hline \multirow[t]{6}{*}{ Age (years) } & $18-24$ & 139 & 23.7 \\
\hline & $25-34$ & 159 & 27.1 \\
\hline & $35-44$ & 128 & 21.8 \\
\hline & $45-64$ & 133 & 22.7 \\
\hline & $65+$ & 28 & 4.8 \\
\hline & & $\begin{array}{l}\text { Mean } \\
35.76\end{array}$ & $\begin{array}{l}\text { SD } \\
13.8\end{array}$ \\
\hline \multirow[t]{4}{*}{ Marital status } & Never married & 130 & 22.1 \\
\hline & Married & 427 & 72.7 \\
\hline & Divorced & 12 & 2.0 \\
\hline & Widowed & 18 & 3.1 \\
\hline \multirow[t]{2}{*}{ Residence } & Urban & 182 & 31.0 \\
\hline & Rural & 405 & 69.0 \\
\hline \multirow[t]{4}{*}{ Educational level } & Cannot read and write & 154 & 26.2 \\
\hline & $\begin{array}{l}\text { Literate, but no formal } \\
\text { education }\end{array}$ & 77 & 13.1 \\
\hline & Primary school & 205 & 34.9 \\
\hline & High school or above & 152 & 25.9 \\
\hline \multirow[t]{2}{*}{ Religion } & Christian & 559 & 95.2 \\
\hline & Muslim & 28 & 4.8 \\
\hline \multirow[t]{7}{*}{ Occupation } & Paid work & 62 & 10.6 \\
\hline & Private work & 66 & 11.2 \\
\hline & Farming & 226 & 38.5 \\
\hline & Housewife & 154 & 26.2 \\
\hline & Student & 53 & 9.0 \\
\hline & Unemployed & 10 & 1.7 \\
\hline & Other & 16 & 2.7 \\
\hline
\end{tabular}




\begin{tabular}{|c|c|c|c|}
\hline \multirow[t]{5}{*}{ Income level or relative wealth } & Very low & 48 & 8.2 \\
\hline & Lower & 195 & 33.2 \\
\hline & Average & 321 & 54.7 \\
\hline & Higher & 21 & 3.6 \\
\hline & Very high & 2 & 0.3 \\
\hline \multirow[t]{2}{*}{ Do you have children? } & No & 152 & 25.9 \\
\hline & Yes & 435 & 74.1 \\
\hline \multirow[t]{3}{*}{ Number of children $(n=435)$} & 1-3 children & 172 & 39.5 \\
\hline & 4-6 children & 192 & 44.1 \\
\hline & More than 6 children & 71 & 16.3 \\
\hline \multirow[t]{8}{*}{ What is the main reason for your visit today? } & New illness & 435 & 74.1 \\
\hline & Acute illness & 39 & 6.6 \\
\hline & Injury & 2 & 0.3 \\
\hline & $\begin{array}{l}\text { Check-up or other preventive } \\
\text { care }\end{array}$ & 4 & 0.7 \\
\hline & Prenatal check-up & 3 & 0.5 \\
\hline & $\begin{array}{l}\text { Follow-up appointment for } \\
\text { earlier chronic illness }\end{array}$ & 94 & 16.0 \\
\hline & $\begin{array}{l}\text { Follow-up appointment for } \\
\text { earlier accident }\end{array}$ & 3 & 0.5 \\
\hline & Other & 7 & 1.2 \\
\hline \multirow{2}{*}{$\begin{array}{l}\text { Have you ever come to this facility or gone to other } \\
\text { places for seeking help? }(n=584)\end{array}$} & No & 427 & 73.1 \\
\hline & Yes & 157 & 26.9 \\
\hline
\end{tabular}




\begin{tabular}{|ll|}
\hline \multicolumn{1}{|c|}{ Item } & Factor loading \\
\hline PHQ-9 & \\
\hline Little interest or pleasure in doing things & 0.74 \\
\hline Feeling down, depressed, or hopeless & 0.79 \\
\hline Sleep problem & 0.73 \\
\hline Feeling tired or having little energy & 0.70 \\
\hline Eating problem & 0.67 \\
\hline Feeling bad about yourself & 0.74 \\
\hline Trouble concentrating & 0.69 \\
\hline Agitation or retardation & 0.72 \\
\hline Thoughts that you would be better off dead & 0.65 \\
\hline PHQ-11 & \\
\hline Little interest or pleasure in doing things & 0.72 \\
\hline Feeling down, depressed, or hopeless & 0.77 \\
\hline Sleep problem & 0.74 \\
\hline Feeling tired or having little energy & 0.68 \\
\hline Eating problem & 0.66 \\
\hline Feeling bad about yourself & 0.73 \\
\hline Trouble concentrating & 0.68 \\
\hline Agitation or retardation & 0.69 \\
\hline Thoughts that you would be better off dead & 0.64 \\
\hline Irritability & 0.65 \\
\hline Noise intolerance & 0.65 \\
\hline WHO-5 & 0.87 \\
\hline Cheerful and good spirit & \\
\hline Calm and relaxed & \\
\hline Active vigorous & 0.87 \\
\hline Fresh and rested & \\
\hline Interest & \\
\hline
\end{tabular}


The PHQ-15 was slightly different as there is a gender specific item (menstrual pain). First a factor analysis including all of the 15 items (PHQ-15a) resulted in three factors with eigenvalue $>1$. The first factor explained $30.8 \%$ and the other two factors explained $7.8 \%$ and $7.0 \%$ of the total variance. Most of the items clearly loaded onto their respective factors, with factor loadings ranging from 0.49 to 0.75 (Table 3). Two items (dizziness and fainting spells), cross-loaded onto two factors. It was generally very difficult to interpret the three factors as items loaded in each of the factors were mixed.

Factor analysis was performed again with the 13 items (PHQ-15b), excluding the gender specific item (Menstrual cramps or other problems with periods) and another item related to sexual intercourse (Pain or problems during sexual intercourse). The data for PHQ-15b (with the 13 items) seemed to suggest bifactorial structure. The first factor explained $34.1 \%$ and the second factor $8.5 \%$ of the total variance. Seven items loaded onto the first factor, with factor loadings ranging from 0.42 to 0.71 , and six items loaded onto the second factor, with factor loadings from 0.54 to 0.66 (Table 4). There were no items which cross-loaded onto more than one factor. It appeared that items loaded onto the second factor were purely physical (e.g. stomach pain, back pain and problems with bowels); whereas factors loaded onto the first factor were not purely physical although they have physical manifestations (e.g. dizziness, trouble sleeping, tiredness, fainting and shortness of breath).

Internal consistency of items, as evaluated by Cronbach's alpha, for all the scales was good: 0.87 for PHQ-9, 0.89 for PHQ-11, 0.83 for PHQ-15 and 0.89 for WHO-5. Internal consistency for the PHQ-2 was lower $(a=0.72)$.

Table 3: Rotated factor loadings of the exploratory factor analysis of PHQ-15a and PHQ-15b 


\begin{tabular}{|c|c|c|c|c|c|}
\hline \multirow[t]{3}{*}{ Item } & \multicolumn{3}{|c|}{ Factor loading } & & \\
\hline & \multirow[b]{2}{*}{$\begin{array}{l}\text { Factor } \\
1\end{array}$} & \multicolumn{2}{|c|}{ PHQ-15a } & \multicolumn{2}{|c|}{ PHQ-15b } \\
\hline & & $\begin{array}{l}\text { Factor } \\
2\end{array}$ & $\begin{array}{l}\text { Factor } \\
3\end{array}$ & $\begin{array}{l}\text { Factor } \\
1\end{array}$ & $\begin{array}{l}\text { Factor } \\
2\end{array}$ \\
\hline Stomach pain & & 0.69 & & & 0.66 \\
\hline Back pain & & 0.54 & & & 0.61 \\
\hline Pain in your arms, legs, or joints & 0.52 & & & & 0.46 \\
\hline $\begin{array}{l}\text { Menstrual cramps or other problems with } \\
\text { periods }\end{array}$ & & & 0.70 & & \\
\hline Headaches & & 0.60 & & & 0.54 \\
\hline Chest pain & 0.57 & & & 0.42 & \\
\hline Dizziness & & 0.42 & 0.45 & 0.55 & \\
\hline Fainting spells & 0.48 & & 0.52 & 0.71 & \\
\hline Heart pound or race & 0.75 & & & 0.69 & \\
\hline Shortness of breath & 0.70 & & & 0.68 & \\
\hline Pain or problems during sexual intercourse & 0.49 & & & & \\
\hline Constipation, loose bowels & & 0.61 & & & 0.60 \\
\hline Nausea & & 0.61 & & & 0.64 \\
\hline Tired or having low energy & 0.61 & & & 0.64 & \\
\hline Trouble sleeping & 0.47 & & & 0.46 & \\
\hline
\end{tabular}

PHQ-15= Patient health questionnaire-15

Concurrent validity and convergent validity

Scores for all of the depression screening scales were moderately or highly correlated with each other (Rho= 0.58 to 0.98 ). The scores for PHQ-2, PHQ-9 and PHQ-11 were highly correlated (Rho= 0.85 to 0.98 ) with each other suggesting one could substitute the other. Scores for all the depression screening scales were moderately correlated with GAD-7 (Rho $=0.55$ to 0.73 ) and WHODAS-2 scores ( 0.58 to 0.74$)$. Scores for all of the depression screening scales were correlated with scores for social support and list of threatening events; however, the correlations were lower ( $R h o=0.21$ to 0.42 ). See Table 5. Differences between psychiatrist depression diagnosed and non-diagnosed cases in the median scores of all the screening scales were statistically significant $(\mathrm{P}<0.01)$. The median score for PHQ-2, PHQ-9, PHQ-11, PHQ-15 and WHO-5 in the diagnosed cases were $3,12,15,11$ and 6 , respectively; whereas in the nondiagnosed cases were $2,7,8,8$ and 9, respectively. 
Table 4: Inter-correlation of depression screening scales and their correlation with anxiety, disability, social support and list of threatening events

\begin{tabular}{|lllllllllll|}
\hline & & $\mathbf{1}$ & $\mathbf{2}$ & $\mathbf{3}$ & $\mathbf{4}$ & $\mathbf{5}$ & $\mathbf{6}$ & $\mathbf{7}$ & $\mathbf{8}$ & $\mathbf{9}$ \\
\hline PHQ-2 & $(1)$ & 1.00 & & & & & & & & \\
\hline PHQ-9 & $(2)$ & 0.87 & 1.00 & & & & & & & \\
\hline PHQ-11 & $(3)$ & 0.85 & 0.98 & 1.00 & & & & & & \\
\hline PHQ-15 & $(4)$ & 0.61 & 0.74 & 0.75 & 1.00 & & & & & \\
\hline WHO-5 & $(5)$ & -0.58 & -0.69 & -0.70 & -0.60 & 1.00 & & & & \\
\hline GAD-7 & $(6)$ & 0.63 & 0.71 & 0.73 & 0.64 & -0.55 & 1.00 & & & \\
\hline WHODAS-2.0 & $(7)$ & 0.65 & 0.74 & 0.74 & 0.69 & -0.58 & 0.70 & 1.00 & & \\
\hline OSSS-3 & $(8)$ & -0.31 & -0.27 & -0.27 & -0.28 & 0.21 & -0.33 & -0.37 & 1.00 & \\
\hline LTE & $(9)$ & 0.38 & 0.40 & 0.42 & 0.37 & -0.34 & 0.43 & 0.41 & -0.27 & 1.00 \\
\hline
\end{tabular}

$P H Q=$ Patient health questionnaire; WHO-5= World Health Organization-Five Well-being Index; GAD$7=$ seven item generalized anxiety disorder scale; WHODAS= World Health Organization Disability Assessment Schedule; OSSS-3= Oslo social support scale; LTE= List of threatening experiences

Association of ratings of items in screening tools with depression diagnosis

All of the PHQ-9 items were significantly associated with depression diagnosis (Table 5). However, items Feeling down, depressed or hopeless; Sleep problem; Feeling bad about oneself and Suicidal ideation were highly associated compared to the other items. The new items we added into the PHQ-9 (Irritability and Noise intolerance) had higher association than all of the PHQ-9 items, except the item Feeling down, depressed and hopeless. Although most of the PHQ-15 items had statistically significant association with depression diagnosis, the strength of association for most of the items was weak. Nevertheless, four items (Fainting spells, Problems during sexual intercourse, Feeling tired or having low energy and Trouble sleeping) had higher association compared to the other items. Overall, it appeared that items measuring emotional problems were highly associated with depression diagnosis more than items measuring cognitive or somatic symptoms. Particularly, items measuring physical problems had weak association with psychiatrist diagnosis of depression (Table 5). All items in the WHO-5 had negative and statistically significant association with depression diagnosis.

Table 5: Association of items in screening tools with depression diagnosis 


\begin{tabular}{|c|c|c|}
\hline Item & Odds Ratio (95\% Cl) & P-value \\
\hline \multicolumn{3}{|l|}{ PHQ-9+2 } \\
\hline Little interest or pleasure in doing things & $1.30(1.05,1.61)$ & $<0.05$ \\
\hline Feeling down, depressed, or hopeless & $2.46(1.87,3.23)$ & $<0.01$ \\
\hline Sleep problem & $2.03(1.55,2.67)$ & $<0.01$ \\
\hline Feeling tired or having little energy & $1.63(1.28,2.08)$ & $<0.01$ \\
\hline Eating problem & $1.40(1.08,1.81)$ & $<0.01$ \\
\hline Feeling bad about yourself & $2.01(1.56,2.59)$ & $<0.01$ \\
\hline Trouble concentrating & $1.97(1.51,2.57)$ & $<0.01$ \\
\hline Agitation or retardation & $1.90(1.43,2.51)$ & $<0.01$ \\
\hline Thoughts that you would be better off dead & $2.04(1.49,2.79)$ & $<0.01$ \\
\hline Irritability & $2.64(1.47,4.72)$ & $<0.01$ \\
\hline Noise intolerance & $2.25(1.30,3.91)$ & $<0.01$ \\
\hline \multicolumn{3}{|l|}{ PHQ-15 } \\
\hline Stomach pain & $1.53(1.10,2.02)$ & $<0.05$ \\
\hline Back pain & $1.17(0.85,1.61)$ & 0.339 \\
\hline Pain in your arms, legs, or joints & $1.50(1.10,2.05)$ & $<0.05$ \\
\hline Menstrual cramps or other problems with periods & $1.31(0.86,1.99)$ & 0.210 \\
\hline Headaches & $1.53(1.06,2.20)$ & $<0.05$ \\
\hline Chest pain & $1.39(0.96,2.02)$ & 0.079 \\
\hline Dizziness & $1.91(1.32,2.75)$ & $<0.05$ \\
\hline Fainting spells & $2.20(1.20,4.04)$ & $<0.05$ \\
\hline heart pound or race & $1.98(1.39,2.84)$ & $<0.01$ \\
\hline Shortness of breath & $1.86(1.30,2.66)$ & $<0.01$ \\
\hline Pain or problems during sexual intercourse & $2.08(1.33,3.24)$ & $<0.01$ \\
\hline Constipation, loose bowels & $1.17(0.80,1.70)$ & 0.423 \\
\hline Nausea, gas or indigestion & $1.52(1.10,2.12)$ & $<0.05$ \\
\hline Feeling tired or having low energy & $2.08(1.41,3.07)$ & $<0.01$ \\
\hline Trouble sleeping & $2.26(1.59,3.23)$ & $<0.01$ \\
\hline
\end{tabular}




\begin{tabular}{|lll|} 
WHO-5 & & \\
\hline Cheerful and good spirit & $0.60(0.48,0.75)$ & $<0.01$ \\
\hline Calm and relaxed & $0.56(0.45,0.71)$ & $<0.01$ \\
Active vigorous & $0.52(0.40,0.67)$ & $<0.01$ \\
Fresh and rested & $0.698(0.58,0.85)$ & $<0.01$ \\
\hline Interest & $0.58(0.45,0.74)$ & $<0.01$ \\
\hline
\end{tabular}

$\mathrm{PHQ}=$ Patient health questionnaire; WHO-5= World Health Organization-Five Well-being Index; $\mathrm{Cl}=$ Confidence interval

\section{Discussion}

In this validation study of depression screening questionnaires in the PHC setting, PHQ-9, PHQ-11 and WHO- 5 were found to be unidimensional and each item in all these scales highly loaded onto the resulting factor, suggesting that these instruments have good construct validity. A systematic review of the psychometric properties of the PHQ-9 [54] showed a good fit for both one factor and two factors solutions. Nevertheless, the one factor model is found to be more parsimonious. Exploratory factor analysis of the PHQ-9 in a PHC setting in rural Ethiopia [18] found that its internal structure is unidimensional, and all items loaded on to the resulting factor with item-factor correlation $>0.35$. The addition of two new items (irritability and noise intolerance) into the PHQ-9 did not change the structure of the scale, suggesting the utility and consistency of these items with the rest of the items. In the Ethiopian socio-cultural context, irritability and noise intolerance are strongly proscribed and usually considered as deviant states [18]. Several previous studies both from high-income countries and LMICs verified a one-factor structure of the WHO-5 through confirmatory factor analysis [42, 55]. A validation study of the Swahili version of the WHO-5 in rural coastal Kenya [43] found a unidimensional structure, and this was maintained across the three study groups (people living with epilepsy, people living with HIV and healthy controls).

PHQ-15 is found to be weak in terms of factor structure. The resulting factors when all items were considered are difficult to inteprete and some of the items cross loaded onto more than one factor. The original PHQ-15 validation study identified three factors when all of the items are considered [29]. However, there are also other studies that suggest bifactorial structure where there is a general somatic factor that all items load to as well as symptom-specific factors [56]. A number of studies, including the original PHQ-15 validation study, have excluded the item related to menstruation problem. In addition, this study has excluded another item (pain during sex) because it has low factor loading. There was similar observation in our data. As a result, the factor analysis was performed again with the 13 items. With the 13 items we found two factors where items clearly loaded onto their respective factors. Nevertheless, studies consistently show that the optimal structure of the PHQ-15 was bifactorial, providing both a 
single global measure of somatization and specific measures of pain, gastrointestinal, cardiopulmonary and fatigue factors [57].

All the depression screening questionnaires were strongly or moderately correlated with each other, suggesting concurrent validity. As expected PHQ-2, PhQ-9 and PHQ-11 were found to be correlated strongly with each other and moderately with the other depression screening questionnaires. PHQ-15 and WHO-5 were associated moderately with the other depression screening questionnaires. All of the depression screening scales were found to have moderate correlation with GAD-7 and WHODAS-2 and weak correlation (but in the expected direction) with social support and LTE, showing that they have good convergent validity. We found statistically significant difference in median scores of all the depression screening tools between psychiatrist diagnosed and non-diagnosed cases, suggesting known group validity of the depression screening questionnaires. This shows that all of the depression screening questionnaires considered in this study can discriminate between two groups known to differ in terms of depression diagnosis. Several previous studies show that both PHQ-9 and PHQ-2 have strong positive association with other depression screening questionnaires [34]. A systematic review study found that the WHO-5 has a very high negative association with self- and observer-administered measures of depressive symptoms [42]. Albeit depression symptoms and GAD-7 scores are different and independent, previous studies show that the two have moderate correlation $[44,46]$. A validation study of both PHQ-9 and PHQ2 in a PHC setting in rural Ethiopia showed that both are highly correlated with WHODAS 2.0 disability score and the number of days of disability in the preceding month [18].

We found that items measuring emotional problems were highly associated with depression diagnosis compared to items measuring somatic symptoms. Items measuring cognitive symptoms were in the middle. This is in contrast to the widely believed assertion that somatic symptoms are important to diagnose depression in non-western socio-cultural contexts. Several studies indicated that depression is least detected in non-western settings, including in $\mathrm{PHC}$, because people in non-western cultures somatize depression [39]. Nevertheless, our study showed that patients who have depression are likely to report emotional and cognitive symptoms more than somatic symptoms. Clinicians are also likely to diagnose depression when patients report more of emotional and cognitive symptoms than somatic symptoms. A previous study conducted in a setting similar to ours [18] showed that although somatic symptoms were the most frequently endorsed symptoms in people with gold standard major depressive disorder, they were less discriminating than items that are more emotional and cognitive in nature. Overall, our study points that brief depression screening questionnaires in LMICs need to focus more on emotional and cognitive symptoms than somatic symptoms in order to increase detection of depression in the PHC setting. We also found that PHQ-15, which is a measure of severity of somatic symptoms [29], functions poorly compared to other measures in terms of construct validity, known group validity and predicting clinician diagnosis of depression. The two items we added to the PHQ-9 (irritability and noise intolerance) were found to be highly associated with clinician diagnosis of depression, suggesting their utility in screening depression in the PHC setting. A previous study pointed the potential utility of irritability as an important mood manifestation of depression in the Ethiopian socio-cultural setting [18]. The item "Little interest or pleasure in doing things" is found to be weak in prediciting diagnosis of depression. Hence, we 
suggest replacing this item with the two new items for screening purposes and even for PHQ after further studies are conducted. The item "Feeling down, depressed, or hopeless" is highly associated with clinician diagnosis of depression, and we suggest including this item for screening.

Our study tried to investigate psychometric properties (concurrent and convergent validity, construct validity and known group validity) of depression screening questionnaires which were not emphasized in previous studies. The other strength of our study is that it recruited a large representative sample in the $\mathrm{PHC}$ setting. We are also able to report the psychometric properties of five depression screening questionnaires (PHQ-2, PHQ-9, PHQ-11, PHQ-15 and WHO-5). However, a few limitations of the study need to be highlighted. First, we did not determine the criterion validity of the measures against a gold standard diagnosis of depression due to design constraints. The study was designed in such a way that clinician diagnosis of depression was done only for those who scored above the cut-off point in one of the measures. Second, we were not able to evaluate the test-retest reliability and responsiveness to change of the measures due to feasibility constraints. Third, the study was facility-based and findings cannot be generalized into the general population. Lastly, we were unable to do confirmatory factor analysis as it was not possible to collect additional data from a separate sample due to feasibility constraints.

\section{Conclusions}

The study demonstrates that the PHQ family of instruments, including the newly expanded version, and WHO- 5 have good psychometric properties for use in a primary care setting. The two symptoms we added to the PHQ-9 are found to be useful to detect depression in the rural Ethiopian setting as they are highly associated with clinician diagnosis of depression. Inclusion of these symptoms also did not change the structure of the PHQ-9. Emotional and cognitive symptoms are found to be more useful than somatic symptoms to predict clinician diagnosis of depression. Hence, there is need to use depression screening scales with focus on emotional and cognitive symptoms to improve the detection of depression in the PHC setting. The study shows that the myth about somatic symptoms being important in LMICs should be reexamined. Future research should focus on determining the criterion validity of PHQ-11 and WHO-5 and the test-retest reliability of all of the depression screening questionnaires considered in this study. Testing the unidimensional structure of PHQ-9, PHQ-11 and WHO-5 using confirmatory factor analysis is warranted.

\section{Abbreviations}

DSM: Diagnostic and Statistical Manual of Mental Disorders Cl: Confidence interval GAD-7: Seven item generalized anxiety disorder scale IDEAS: Improving detection of depression in primary care in SubSaharan Africa LMICs: Low and middle-income countries LTE: List of threatening experiences MINI: MiniInternational Neuropsychiatric Interview OSSS-3: Three item Oslo social support scale PHC: Primary healthcare PHQ: Patient health questionnaire PRIME: Programme for Improving Mental Healthcare Rho: Spearman's rank order correlation coefficient SD: Standard deviation SNPPR: Southern Nations, 
Nationalities and peoples Region WHO: World Health Organization WHO-5: World Health OrganizationFive Well-being Index WHODAS: World Health Organization Disability Assessment Schedule

\section{Declarations}

\section{Acknowledgments}

We are grateful to all the participants for giving their time and the data. We would like to thank field workers and field supervisors for collecting the data. We would also like to acknowledge the UK Medical Research Council (MRC) and the UK Department for International Development (DFID) for funding.

\section{Funding}

This study is jointly funded by the UK Medical Research Council (MRC) and the UK Department for International Development (DFID) under the MRC/DFID Concordant agreement through the Africa Research Leader scheme (Grant Ref: MR/M025470/1).

\section{Availability of data and materials}

The datasets used and/or analyzed during the current study are part of an ongoing study [the IDEAS project] and cannot currently be publicly available [due to project requirements not to make the data publicly available until the forthcoming papers are drafted], but are available from the corresponding author [after the forthcoming papers are drafted] up on reasonable request.

\section{Authors' contributions}

$\mathrm{KH}, \mathrm{AF}, \mathrm{GM}$ conceived and designed the study. RB did coordination of the recruitment and data collection process. $\mathrm{KH}$ did the data analysis and drafted the manuscript. $\mathrm{GM}$ and $\mathrm{CH}$ supported in interpretation of data and contributed in the writing of the manuscript. $\mathrm{CH}, \mathrm{GM}$ and AF critically revised and substantially contributed throughout the writing of the manuscript. All authors approved the final manuscript to be submitted for publication.

\section{Competing interests}

The authors declare that they have no competing interests.

\section{Consent for publication}

$\mathrm{N} / \mathrm{A}$

Ethics approval and consent to participate 
The study was approved by the Institutional Review Board of the College of Health Sciences, Addis Ababa University (Reference Number 007/18/Psy). We confirm that all methods related to the human participants were performed in accordance with the Declaration of Helsinki. Written informed consent was obtained from all the participants after the nature of the study and the information sought had been fully explained.

\section{References}

1. Ferrari AJ, Charlson FJ, Norman RE, Patten SB, Freedman G, Murray CJ, Vos T, Whiteford HA: Burden of depressive disorders by country, sex, age, and year: findings from the global burden of disease study 2010. PLoS medicine 2013, 10(11):e1001547.

2. Mathers CD, Loncar D: Projections of global mortality and burden of disease from 2002 to 2030 . PLoS medicine 2006, 3(11):e442.

3. Vigo D, Thornicroft G, Atun R: Estimating the true global burden of mental illness. The Lancet Psychiatry 2016, 3(2):171-178.

4. Wang PS, Simon G, Kessler RC: The economic burden of depression and the cost-effectiveness of treatment. International journal of methods in psychiatric research 2003, 12(1):22-33.

5. McLaughlin KA: The public health impact of major depression: a call for interdisciplinary prevention efforts. Prevention Science 2011, 12(4):361-371.

6. Januzzi JL, Stern TA, Pasternak RC, DeSanctis RW: The influence of anxiety and depression on outcomes of patients with coronary artery disease. Archives of internal medicine 2000, 160(13):1913-1921.

7. Katon W, Schulberg H: Epidemiology of depression in primary care. General hospital psychiatry 1992, 14(4):237-247.

8. Fekadu A, Medhin G, Selamu M, Giorgis TW, Lund C, Alem A, Prince M, Hanlon C: Recognition of depression by primary care clinicians in rural Ethiopia. BMC family practice 2017, 18(1):1-9.

9. Craven MA, Bland R: Depression in primary care: current and future challenges. The Canadian Journal of Psychiatry 2013, 58(8):442-448.

10. Mitchell AJ, Vaze A, Rao S: Clinical diagnosis of depression in primary care: a meta-analysis. The Lancet 2009, 374(9690):609-619.

11. Udedi M: The prevalence of depression among patients and its detection by primary health care workers at Matawale Health Centre (Zomba). Malawi Med J 2014, 26(2):34-37.

12. Ferenchick EK, Ramanuj P, Pincus HA: Depression in primary care: part 1-screening and diagnosis. BMJ 2019, 365.

13. Thombs BD, Saadat N, Riehm KE, Karter JM, Vaswani A, Andrews BK, Simons P, Cosgrove L: Consistency and sources of divergence in recommendations on screening with questionnaires for presently experienced health problems or symptoms: a comparison of recommendations from the 
Canadian Task Force on Preventive Health Care, UK National Screening Committee, and US Preventive Services Task Force. BMC medicine 2017, 15(1):1-17.

14. Gilbody S, Sheldon T, House A: Screening and case-finding instruments for depression: a metaanalysis. Cmaj 2008, 178(8):997-1003.

15. Romera I, Montejo AL, Aragones E, Angel Arbesu J, Iglesias-Garcia C, Lopez S, Antonio Lozano J, Pamulapati S, Yruretagoyena B, Gilaberte I: Systematic depression screening in high-risk patients attending primary care: a pragmatic cluster-randomized trial. Bmc Psychiatry 2013, 13.

16. Yonkers KA, Smith MV, Lin H, Howell HB, Shao L, Rosenheck RA: Depression screening of perinatal women: an evaluation of the healthy start depression initiative. Psychiatric services 2009, 60(3):322-328.

17. Gilbody SM, House AO, Sheldon TA: Routinely administered questionnaires for depression and anxiety: systematic review. BMJ 2001, 322(7283):406-409.

18. Hanlon C, Medhin G, Selamu M, Breuer E, Worku B, Hailemariam M, Lund C, Prince M, Fekadu A: Validity of brief screening questionnaires to detect depression in primary care in Ethiopia. Journal of affective disorders 2015, 186:32-39.

19. Ali G-C, Ryan G, De Silva MJ: Validated screening tools for common mental disorders in low and middle income countries: a systematic review. PloS one 2016, 11(6):e0156939.

20. El-Den S, Chen TF, Gan Y-L, Wong E, O'Reilly CL: The psychometric properties of depression screening tools in primary healthcare settings: A systematic review. Journal of affective disorders 2018, 225:503-522.

21. Gelaye B, Williams MA, Lemma S, Deyessa N, Bahretibeb Y, Shibre T, Wondimagegn D, Lemenhe A, Fann JR, Vander Stoep A: Validity of the patient health questionnaire-9 for depression screening and diagnosis in East Africa. Psychiatry research 2013, 210(2):653-661.

22. Degefa M, Dubale B, Bayouh F, Ayele B, Zewde Y: Validation of the PHQ-9 depression scale in Ethiopian cancer patients attending the oncology clinic at Tikur Anbessa specialized hospital. $B M C$ psychiatry 2020, 20(1):1-7.

23. Reda AA: Reliability and validity of the Ethiopian version of the hospital anxiety and depression scale (HADS) in HIV infected patients. PLoS One 2011, 6(1):e16049.

24. Fekadu A, Medhin G, Selamu M, Hailemariam M, Alem A, Giorgis TW, Breuer E, Lund C, Prince M, Hanlon C: Population level mental distress in rural Ethiopia. BMC psychiatry 2014, 14(1):1-13.

25. Habtamu K, Medhin G, Selamu M, Tirfessa K, Hanlon C, Fekadu A: Functional impairment among people diagnosed with depression in primary healthcare in rural Ethiopia: a comparative crosssectional study. International journal of mental health systems 2019, 13(1):1-12.

26. Fekadu A, Hanlon C, Medhin G, Alem A, Selamu M, Giorgis TW, Shibre T, Teferra S, Tegegn T, Breuer E: Development of a scalable mental healthcare plan for a rural district in Ethiopia. The British journal of psychiatry 2016, 208(s56):s4-s12.

27. Mayston R, Frissa S, Tekola B, Hanlon C, Prince M, Fekadu A: Explanatory models of depression in sub-Saharan Africa: Synthesis of qualitative evidence. Social science \& medicine 2020, 246:112760. 
28. Kroenke K, Spitzer RL, Williams JB: The PHQ-9: validity of a brief depression severity measure. Journal of general internal medicine 2001, 16(9):606-613.

29. Kroenke K, Spitzer R, Williams J: The PHQ-15: Validity of a new measure forevaluating somatic symptom severity. Psychosom Med 2002, 64:258-266.

30. Bech P, Olsen LR, Kjoller M, Rasmussen NK: Measuring well-being rather than the absence of distress symptoms: a comparison of the SF-36 Mental Health subscale and the WHO-Five well-being scale. International journal of methods in psychiatric research 2003, 12(2):85-91.

31. Spitzer RL, Williams JB, Kroenke K, Hornyak R, McMurray J, Group PHQO-GS: Validity and utility of the PRIME-MD patient health questionnaire in assessment of $\mathbf{3 0 0 0}$ obstetric-gynecologic patients: the PRIME-MD Patient Health Questionnaire Obstetrics-Gynecology Study. American journal of obstetrics and gynecology 2000, 183(3):759-769.

32. Americal Psychiatric Association: Diagnostic and statistical manual of mental disorders. Washington, DC: American Psychiatric Anociation; 1994.

33. Kroenke K, Spitzer RL: The PHQ-9: A new depression diagnostic and severity measure. J Gen Intern Med 2002, 32: 509-515.

34. Ghazisaeedi M, Mahmoodi H, Arpaci I, Mehrdar S, Barzegari S: Validity, Reliability, and Optimal Cutoff Scores of the WHO-5, PHQ-9, and PHQ-2 to Screen Depression Among University Students in Iran. International Journal of Mental Health and Addiction 2021:1-10.

35. Kroenke K, Spitzer RL, Williams JB: The Patient Health Questionnaire-2: validity of a two-item depression screener. Medical care 2003:1284-1292.

36. Spitzer R, Kroenke K, Williams J: Patient Health Questionnaire Primary Care 676 Study G. Validation and utility of a self-report version of PRIME-MD: the PHQ 677 primary care study. Jama 1999, 282(18):1737-1744.

37. Toussaint A, Kroenke K, Baye F, Lourens S: Comparing the Patient Health Questionnaire - 15 and the Somatic Symptom Scale - 8 as measures of somatic symptom burden. Journal of psychosomatic research 2017, 101:44-50.

38. Sitnikova K, Dijkstra-Kersten SMA, Mokkink LB, Terluin B, van Marwijk HWJ, Leone SS, van der Horst $\mathrm{HE}$, van der Wouden JC: Systematic review of measurement properties of questionnaires measuring somatization in primary care patients. Journal of psychosomatic research 2017, 103:42-62.

39. Marsella AJ: Cultural aspects of depressive experience and disorders. Online readings in psychology and culture 2003, 10(2):2307-0919.1081.

40. Lyoo YC, Ju S, Kim E, Kim JE, Lee JH: The patient health questionnaire-15 and its abbreviated version as screening tools for depression in Korean college and graduate students. Comprehensive psychiatry 2014, 55(3):743-748.

41. Krieger T, Zimmermann J, Huffziger S, Ubl B, Diener C, Kuehner C, Holtforth MG: Measuring depression with a well-being index: further evidence for the validity of the WHO Well-Being Index (WHO-5) as a measure of the severity of depression. Journal of affective disorders 2014, 156:240244. 
42. Topp CW, Østergaard SD, Søndergaard S, Bech P: The WHO-5 Well-Being Index: a systematic review of the literature. Psychotherapy and psychosomatics 2015, 84(3):167-176.

43. Chongwo E, Ssewanyana D, Nasambu C, Mwangala PN, Mwangi PM, Nyongesa MK, Newton CR, Abubakar A: Validation of a Swahili version of the World Health Organization 5-item well-being index among adults living with HIV and epilepsy in rural coastal Kenya. Global health research and policy 2018, 3(1):1-7.

44. Spitzer RL, Kroenke K, Williams JB, Löwe B: A brief measure for assessing generalized anxiety disorder: the GAD-7. Arch Intern Med 2006, 166(10):1092-1097.

45. Sapra A, Bhandari $P$, Sharma $S$, Chanpura T, Lopp L: Using generalized anxiety disorder-2 (GAD-2) and GAD-7 in a primary care setting. Cureus 2020, 12(5).

46. Jordan P, Shedden-Mora MC, Löwe B: Psychometric analysis of the Generalized Anxiety Disorder scale (GAD-7) in primary care using modern item response theory. PloS one 2017, 12(8):e0182162e0182162.

47. Üstün TB, Kostanjsek N, Chatterji S, Rehm J: Measuring health and disability: Manual for WHO disability assessment schedule WHODAS 2.0: World Health Organization; 2010.

48. Üstün TB, Chatterji S, Kostanjsek N, Rehm J, Kennedy C, Epping-Jordan J, Saxena S, Korff Mv, Pull C: Developing the World Health Organization disability assessment schedule 2.0. Bulletin of the World Health Organization 2010, 88:815-823.

49. Habtamu K, Alem A, Medhin G, Fekadu A, Dewey M, Prince M, Hanlon C: Validation of the World Health Organization disability assessment schedule in people with severe mental disorders in rural Ethiopia. Health and quality of life outcomes 2017, 15(1):1-11.

50. Bøen $\mathrm{H}$ : Characteristics of senior centre users-and the impact of a group programme on social support and late-life depression. Norsk epidemiologi 2012, 22(2).

51. Dalgard OS, Dowrick C, Lehtinen V, Vazquez-Barquero JL, Casey P, Wilkinson G, Ayuso-Mateos JL, Page H, Dunn G: Negative life events, social support and gender difference in depression. Social psychiatry and psychiatric epidemiology 2006, 41(6):444-451.

52. Brugha T, Bebbington $P$, Tennant $C$, Hurry J: The List of Threatening Experiences: a subset of 12 life event categories with considerable long-term contextual threat. Psychological medicine 1985, 15(1):189-194.

53. Weobong B, Akpalu B, Doku V, Owusu-Agyei S, Hurt L, Kirkwood B, Prince M: The comparative validity of screening scales for postnatal common mental disorder in Kintampo, Ghana. Journal of affective disorders 2009, 113(1-2):109-117.

54. Lamela D, Soreira C, Matos P, Morais A: Systematic review of the factor structure and measurement invariance of the patient health questionnaire-9 (PHQ-9) and validation of the Portuguese version in community settings. Journal of Affective Disorders 2020, 276:220-233.

55. Hajos TR, Pouwer F, Skovlund S, Den Oudsten BL, Geelhoed-Duijvestijn P, Tack C, Snoek FJ: Psychometric and screening properties of the WHO-5 well-being index in adult outpatients with Type 1 or Type 2 diabetes mellitus. Diabetic Medicine 2013, 30(2):e63-e69. 
56. Witthöft M, Hiller W, Loch N, Jasper F: The latent structure of medically unexplained symptoms and its relation to functional somatic syndromes. International journal of behavioral medicine 2013, 20(2):172-183.

57. Cano-García FJ, Muñoz-Navarro R, Abad AS, Moretti LS, Medrano LA, Ruiz-Rodríguez P, GonzálezBlanch C, Moriana JA, Cano-Vindel A: Latent structure and factor invariance of somatic symptoms in the patient health questionnaire (PHQ-15). Journal of affective disorders 2020, 261:21-29.

\section{Figures}

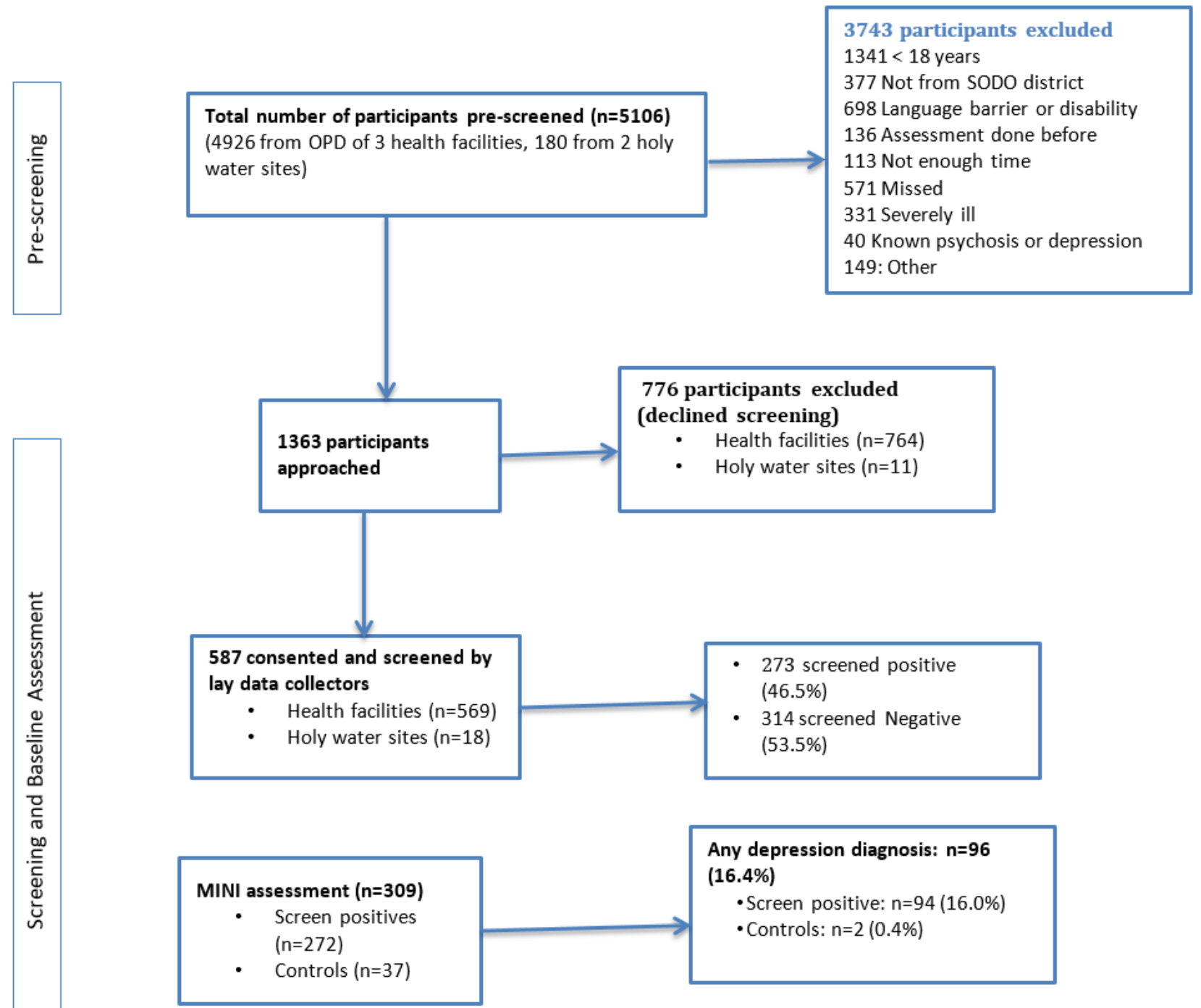

Figure 1

Participant recruitment flow chart 